\title{
Exemples d'études de développabilité apportant un éclairage à la prise de décision
}

> De nos jours, la génération d'anticorps thérapeutiques doit être plus rapide avec des coûts de développement moins importants. Pour cela, des prédictions in silico sont associées à des technologies de criblage et de caractérisation de pointe. Les exemples choisis ici sont nonexhaustifs mais illustrent ce besoin de travailler en parallèle. <
De nos jours, la plupart des anticorps monoclonaux thérapeutiques générés dans l'industrie pharmaceutique sont des anticorps humains; il est de plus en plus rare d'avoir recours au processus d'humanisation. Certains anticorps, pour devenir des anticorps thérapeutiques, peuvent nécessiter ce savoir-faire ; il s'agit de modifier les séquences de l'anticorps par un ensemble de mutations afin d'obtenir des séquences dont le taux d'identité est le plus proche possible des lignées germinales humaines. Au sein de l'ensemble des variants réalisés, l'anticorps lead sera sélectionné.

\section{Cas nécessitant une humanisation}

Dans cet exemple, un ensemble de mutations dans les domaines variables des chaînes lourdes (VH) et légères (VL) de l'anticorps lead ( $\mathrm{mAbl}$ ) ont été réalisées à partir de modélisations in silico, en prenant soin de conserver les CDR de l'anticorps parental (Tableau I).

Nous avons pu confirmer que les variants (mAb2 à mAb6) conservent bien les mêmes caractéristiques de fixation à l'antigène que l'anticorps parental ainsi que ses fonctions biologiques.

Les variants du mAbl montrent des niveaux d'expression augmentés, des températures de fusion ( $\mathrm{Tm}$ ) et des Tonset inférieures à celles de l'anticorps parental : la Tm du Fab d'un anticorps est un meilleur indicateur de stabilité que le Tm correspondant à la région Fc [1]. À noter que les mutations du Fab n'influencent pas, ici, cette caractéristique. II est alors possible de classer les variants mAb2 à mAb6 sur la base de ces critères (Tableau II).
Jacques Dumas ${ }^{1}$, Anne Sévérac ${ }^{1}$, Cendrine Lemoine ${ }^{1}$, Sylvain Huille ${ }^{2}$, Alexey Rak ${ }^{3}$, Catherine Prades ${ }^{1}$

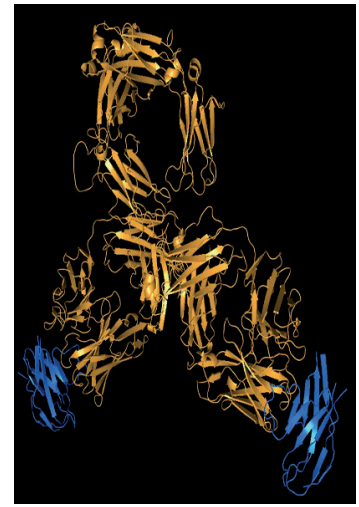

${ }^{1}$ Biologics Research/Sanofi R\&D, 13 quai Jules Guesde, 94403 Vitry-sur-Seine, France. ${ }^{2}$ Biologics Drug Products Development/Sanofi R\&D, 13 quai Jules Guesde, 94403 Vitry-sur-seine, France. ${ }^{3}$ Integrated Drug Discovery Sanofi, 13 quai Jules Guesde, 94403 Vitry-sur-Seine, France. Catherine.Prades@sanofi.com

\begin{tabular}{lll}
\hline Variants & mAb1_VH & mAb 1_VL \\
\hline VHIA & 15 mutations & \\
\hline VHIB & 11 mutations & \\
\hline VLIA & & 17 mutations \\
\hline VLIB & 15 mutations \\
\hline
\end{tabular}

Tableau I. Nombre de mutations à effectuer dans les domaines VH et VL de chaînes lourdes et légères $d$ 'après les prédictions in silico.

Lorsque nous examinons ce qu'il se passe lors d'expériences de stress de température ( 2 semaines à une température de $40^{\circ} \mathrm{C}$ ), nous n'observons pas de changement, ce qui montre que ces variants sont globalement stables. Une méthode orthogonale par microcalorimétrie vient confirmer ces observations [2]. Cette notion de croisement d'observations par plusieurs méthodes analytiques est importante pour avoir un niveau de confiance suffisant dans les observations.

De plus, il convient d'utiliser aussi des méthodes basées sur la DLS (dynamic light scattering) [3] qui montrent dans le cas présent que ces variants se répartissent avec des rayons hydrodynamiques comparables avant et après stress, avec une polydispersité basse et aucun nouveau pic.

Le stress de température n'affecte pas non plus l'affinité mesurée par SPR (surface plasmon resonance), ce qu'il convient de toujours vérifier. $\varepsilon$ n revanche, les légères différences de $K D$ entre ces variants peuvent aussi guider le choix du lead, dans la mesure où une des 


\begin{tabular}{|c|c|c|c|c|c|c|}
\hline & mAb 1 & mAb2 & mAb3 & mAb4 & mAb5 & mAb6 \\
\hline Cellule hôte & CHO & CHO & CHO & CHO & CHO & CHO \\
\hline Rendement/titre (pg/cellule/jour) & ND & 0,62 & 2,16 & 2,6 & 2,35 & 1,57 \\
\hline Interaction antigène-anticorps (SPR) & Confirmée & Confirmée & Confirmée & Confirmée & Confirmée & Confirmée \\
\hline Pureté (après Protéine A par SEC) & ND & $98,40 \%$ & $98,80 \%$ & $99,40 \%$ & $98,70 \%$ & $99,50 \%$ \\
\hline Profil de masse (HMWs + LMWs par SEC) & $0,7 \%+1,2 \%$ & $0,2 \%+1,4 \%$ & $1,0 \%+0,1 \%$ & $0,5 \%+0,1 \%$ & $1,2 \%+0,1 \%$ & $0,4 \%+0,1 \%$ \\
\hline $\begin{array}{l}\text { Stabilité thermique par nano DSF (onset } \\
\text { température) }\end{array}$ & $63,9^{\circ} \mathrm{C}$ & $46,4^{\circ} \mathrm{C}$ & $47,4^{\circ} \mathrm{C}$ & $44,8^{\circ} \mathrm{C}$ & $46,1^{\circ} \mathrm{C}$ & $43,6^{\circ} \mathrm{C}$ \\
\hline Stabilité thermique par nano DSF (Fab Tm) & $82,8^{\circ} \mathrm{C}$ & $82,1^{\circ} \mathrm{C}$ & $80,9^{\circ} \mathrm{C}$ & $82,7^{\circ} \mathrm{C}$ & $77,4^{\circ} \mathrm{C}$ & $79,5^{\circ} \mathrm{C}$ \\
\hline Intégrité (SM) & masse attendue & masse attendue & masse attendue & masse attendue & masse attendue & masse attendue \\
\hline
\end{tabular}

Tableau II. Données expérimentales de développabilité de différents variants d'anticorps. SEC : size exclusion chromatography ; HMWs : high molecular weights; LMWs : low molecular weights : SPR : surface plasmon resonance; Tm : température de fusion; SM : spectrométrie de masse.

méthodes de mesure de la fixation de l'anticorps sur l'antigène par ELISA (enzyme-linked immunosorbent assay) et une deuxième par cytométrie de flux sur une lignée murine exprimant l'antigène humain ne montrent pas de différence significative dans les $\varepsilon C_{50}$ mesurées. La spectrométrie de masse est utilisée, afin d'analyser si les stress combinés de $\mathrm{pH}$ et de température accélèrent des instabilités potentielles (intégrité, dégradations), tout comme l'analyse chromatographique par exclusion de taille est utilisée pour analyser l'éventuelle apparition d'entités de hautes et basses masses moléculaires.

C'est la combinaison des résultats de ces tests analytiques qui permet aux équipes de recherche de donner les indications qui participeront au choix de candidats les plus favorables au développement.

\section{Cas de minimisation d'un risque d'immunogénicité et modifications chimiques de motifs problématiques : limites d'une approche qui ne serait basée que sur le « in silico »}

Le risque d'immunogénicité chez le patient est très difficile à prédire, malgré les efforts énormes consentis par des consortiums européens tels que I'IMI «ABIRISK» (anti-biopharmaceutical immunization: prediction and analysis of clinical relevance to minimize the risk). $\varepsilon n$ effet, les tests cellulaires tout comme ceux réalisés chez l'animal présentent certaines limitations. La FDA (food and drug administratio) demande néanmoins qu'une évaluation du risque soit réalisée afin de minimiser les risques tels que l'induction d'ADA (anti-drug antibodies) qui peut parfois réduire notablement l'efficacité du produit, voire générer de sévères réactions chez le patient.

Pour illustrer la difficulté que présente une tentative de « dé-immunisation » (abolir le risque d'immunogénicité de l'anticorps injecté en diminuant le nombre d'épitopes T prédits), le cas d'anticorps obtenus chez des souris dont le répertoire lymphocytaire n'est pas humanisé, est un bon exemple.

Quels que soient les résultats des prédictions in silico, ceci ne permet pas d'exclure tout risque d'immunogénicité. Si nous partons du principe que l'humanisation diminue les risques d'induction d'ADA, pour ce faire, il est nécessaire de déterminer quels acides aminés peuvent être modifiés par modélisation in silico, dans ce cadre. Le processus d'humanisation ne doit pas introduire d'épitopes T, qui deviendraient alors potentiellement responsables de nouveaux risques immunogéniques. Une fois l'analyse réalisée grâce à un ensemble de modèles, le résultat obtenu dans l'exemple choisi a amené à élaborer deux stratégies de travail : soit effectuer une humanisation en tenant compte des épitopes $T$ et des motifs problématiques à risque, soit effectuer les deux tâches séquentiellement.

Dans le cadre d'une approche combinant «humanisation et dé-immunisation », nous nous trouvons confrontés à un problème de taille : il nous faut générer selon le nombre de mutations proposées, entre 320 et 20420 mutants, dans la mesure où il nous faut consi-

\begin{tabular}{lccccccc}
\hline & \multicolumn{5}{c}{$\begin{array}{c}\text { Nombre de mutations } \\
\text { AA indésirables }\end{array}$} & \multicolumn{1}{c}{ proposées } & Scénario \\
\cline { 2 - 7 } & & A & B & C & D & $\boldsymbol{\varepsilon}$ \\
\hline HC-CDR1 & DY & 3 & 1 & 0 & 0 & 0 \\
\hline HC-CDR2 & DP & 3 & 2 & 2 & 2 & 1 \\
\hline & NG & 3 & 2 & 2 & 2 & 1 \\
\hline LC-CDR1 & NT & 3 & 2 & 2 & 1 & 1 \\
\hline Nb total de variants & 20480 & 3240 & 1620 & 1080 & 320 \\
\hline
\end{tabular}

Tableau III. Nombre de mutations à tester selon les prédictions in silico d'acides aminés indésirables. 


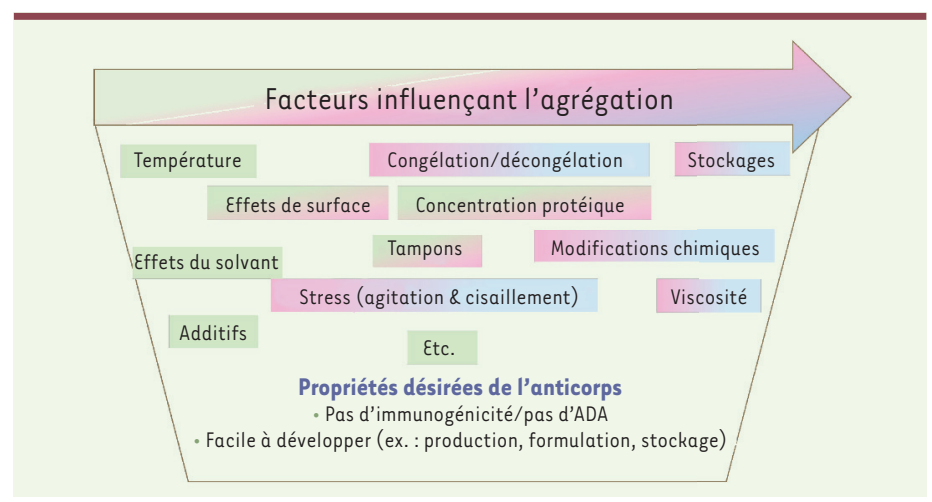

Figure 1. Facteurs influençant l'agrégation.

dérer 20 variations pour l'humanisation, combinées aux motifs non souhaités (Tableau III). Si la version basse du nombre de mutations à effectuer est retenue, le risque de perdre la liaison à l'antigène cible est considérablement accru. La version haute du nombre de mutations occasionne des temps et des coûts liés à l'expérimentation trop élevés. L'approche séquentielle en deux étapes (humaniser, puis ôter les motifs non souhaités) permet de réduire le nombre de variants à tester à 20 à la première étape puis de générer 15 variants pour les motifs problématiques. Cette approche, même si elle est significativement moins coûteuse est beaucoup plus longue. Cependant, le risque de perdre la liaison à l'antigène est minimisé.

Générer directement des anticorps humains permet d'éviter que l'étape d'humanisation complexifie l'étape de développabilité, et le risque d'avoir trop de variants à réaliser et donc de ne pouvoir ainsi contrôler les délais et les coûts.

\section{Cas d'une agrégation de l'anticorps}

Le risque d'agrégation d'un anticorps est sans nul doute une des pires craintes au cours du processus de fabrication et de contrôle (CMC) de la molécule, d'autant que, la plupart du temps, il est nécessaire d'utiliser des concentrations d'anticorps très élevées pour réduire les volumes injectés aux patients. Le problème est d'autant plus épineux au stade Recherche que la quantité d'anticorps purifié est alors faible. Même si les technologies d'analyse sont de plus en plus nombreuses et sophistiquées et de moins en moins consommatrices de protéine, la miniaturisation reste donc un défi majeur. Les facteurs qui influencent l'agrégation sont très nombreux et dépassent largement la question de la concentration (Figure 1). Connaître les seuils de tolérance de l'anticorps est donc primordial, que ce soit au cours des différentes étapes de la recherche au développement, puis du développement à la production, et enfin du conditionnement à l'utilisation clinique.

La FDA requiert désormais en plus des spécifications fixées pour les particules de plus de 10 microns et de plus de 25 microns, d'assurer et de documenter les mesures de particules de plus petites tailles supérieures à 1 ou 2 microns, évaluées par une méthode quantitative quelle qu'elle soit, sans préférence à ce jour. Pour pouvoir vendre un produit, ces informations, montrant la caractérisation de ces «subvisible particles» (SvP) et la mise en place d'une

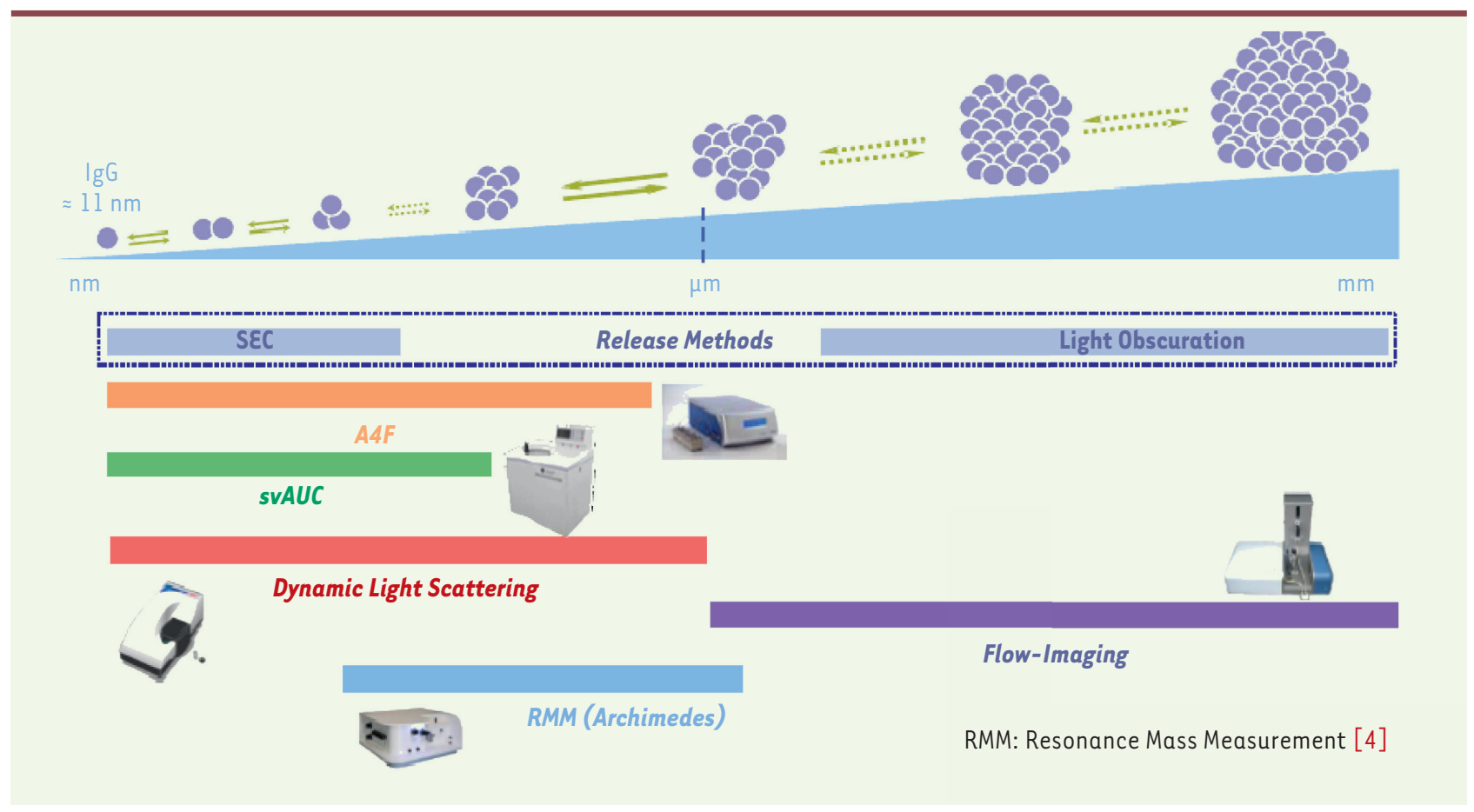

Figure 2. Techniques de mesure de l'agrégation et d'évaluation des particules présentes. 
stratégie de contrôle qualité, sont essentielles pour le dossier d'IND (investigation new drug). Cette question de la présence de particules liées à l'agrégation sera donc examinée lors du Développement, un stade où les productions de l'anticorps atteindront des quantités ne limitant plus les études physiques. Pour mesurer l'agrégation et évaluer les particules présentes, différentes techniques s'offrent à nous (Figure 2). Au stade Recherche, les efforts sont focalisés sur l'agrégation. Les particules visibles seront déterminées ensuite en Développement. La qualité du produit augmente au cours des phases de développement en particulier entre des lots de phase I et phase II pour les agrégats solubles. Dans les phases de recherche, au cours des tests de stress dans différents tampons, il est courant d'observer qu'un $\mathrm{pH}$ est plus défavorable qu'un autre, en générant plus d'agrégats, et que certains variants tolèrent mieux ces variations de $\mathrm{pH}$, même si les hautes et basses masses moléculaires seront conservées.

\section{Conclusion}

Les exemples décrits ci-dessus ne sont qu'une illustration de ce qu'il convient de prendre en compte pour caractériser et sélectionner des candidats thérapeutiques développables. En effet, la génération d'anticorps de haute qualité, obtenus de plus en plus rapidement tout en ayant un coût de développement de moins en moins conséquent, nécessite la combinaison de prédictions in silico alliée à des technologies de criblage et de caractérisation permettant d'évaluer et de valider leur dévelopabilité de manière quasi-simultanée. $\diamond$

\section{SUMMARY}

Developability assessment with case studies highlighting the decision taking

Therapeutic antibodies generation has to be faster with less development costs. This requires combination of in silico predictions associated with cutting edge screening and characterization technologies. Here, nonexhaustive examples illustrate this simultaneity need. $\diamond$

\section{LIENS D'INTÉR $\hat{T}$}

Les auteurs déclarent n'avoir aucun lien d'intérêt concernant les données publiées dans cet article.

\section{RÉFÉRENCES}

1. Largy E, Cantais F, van Vyncht $G$, et al. Orthogonal liquid chromatographymass spectrometry methods for the comprehensive characterization of therapeutic glycoproteins, from released glycans to intact protein level. J Chromatogr A 2017 ; 1498 : 128-46.

2. Vermeer AWP, Norde $W$. The thermal stability of immunoglobulin : unfolding and aggregation of a multi-domain protein. Biophys J $2000 ; 78: 394-404$.

3. Brader ML, Estey T, Bai S, et al. Examination of thermal unfolding and aggregation profiles of a series of developable therapeutic monoclonal antibodies. Mol Pharmaceutics 2015 ; 12 : 1005-17.

4. Patel AR, Lau D, Liu J. Quantification and characterization of micrometer and submicrometer subvisible particles in protein therapeutics by use of a suspended microchannel resonator. Anal Chem 2012 ; 84 : 6833-40.
TIRÉS À PART

C. Prades

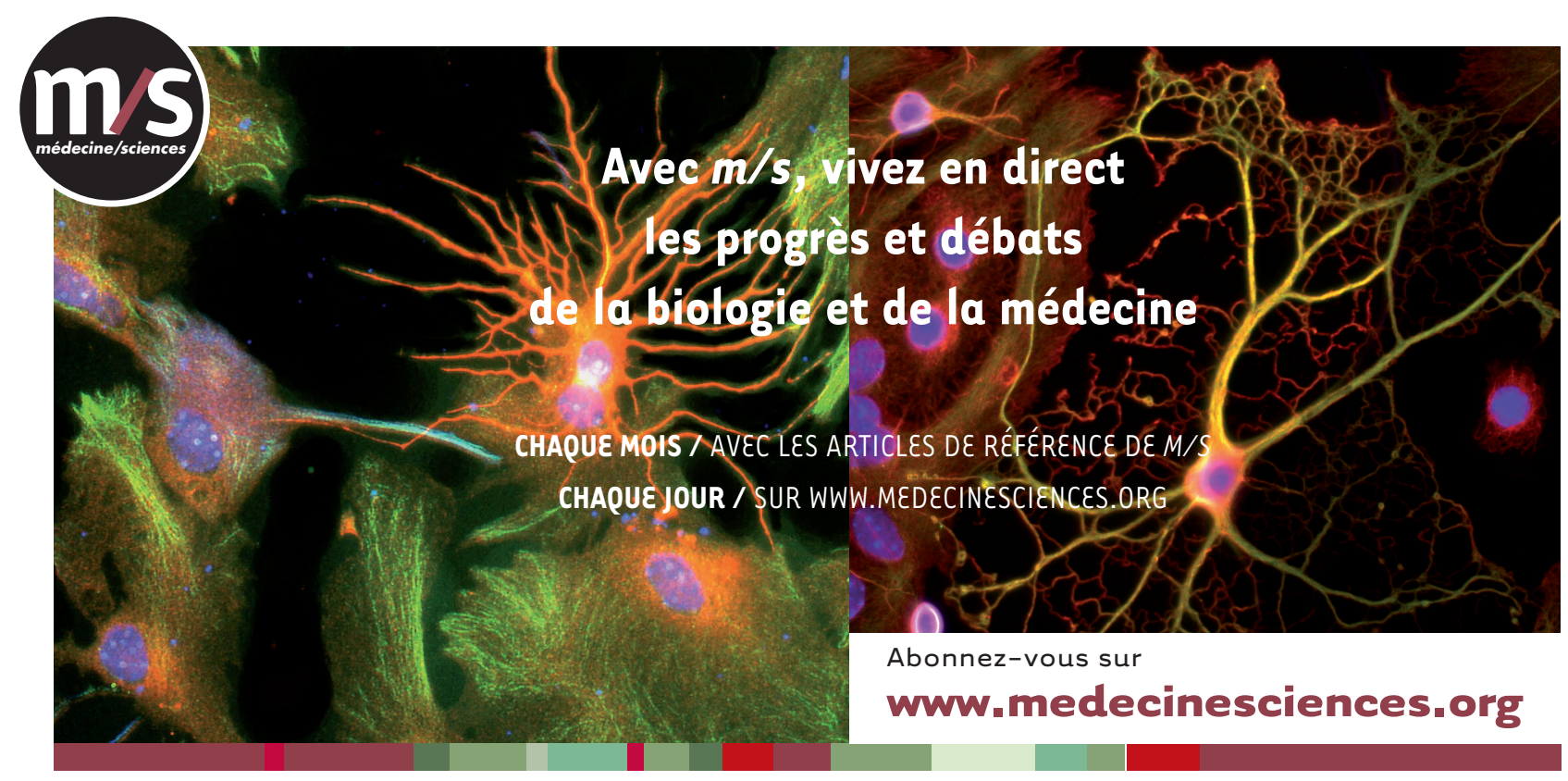

